\title{
Examining the central effects of chronic stressful social isolation on rats
}

\author{
TAHANI K. ALSHAMMARI ${ }^{1}$, HAJAR M. ALGHAMDI ${ }^{2}$, HESSA E. ALDUHAILAN ${ }^{1}$, \\ MAHA F. SAJA $^{3}$, NOUF M. ALRASHEED ${ }^{1}$ and MUSAAD A. ALSHAMMARI ${ }^{1}$ \\ ${ }^{1}$ Department of Pharmacology and Toxicology; ${ }^{2}$ Pharmacology and Toxicology Graduate Program, College of Pharmacy; \\ ${ }^{3}$ Department of Physiology, College of Medicine, King Saud University, Riyadh 11451, Kingdom of Saudi Arabia
}

Received June 11, 2020; Accepted September 16, 2020

DOI: $10.3892 /$ br.2020.1363

\begin{abstract}
Stress-related disorders are extremely complex and current treatment strategies have limitations. The present study investigated alternative pathological mechanisms using a combination of multiple environmental approaches with biochemical and molecular tools. The aim of the present study was to evaluate blood-brain-barrier (BBB) integrity in socially manipulated animal housing conditions. Multiple environmentally-related models were employed in the current study. The main model proposed (chronically isolated rats) was biochemically validated using the level of peripheral corticosterone. The current study examined and compared the mRNA levels of certain inflammatory and BBB markers in the hippocampal tissue of chronically isolated rats, including claudin-5 (cldn5) and tight junction protein (tjp). Animals were divided into four groups: i) Standard housed rats (controls); ii) chronically isolated rats; iii) control rats treated with fluoxetine, which is a standard selective serotonin reuptake inhibitor; and iv) isolated rats treated with fluoxetine. To further examine the effect of environmental conditions on BBB markers, the current study assessed BBB markers in enriched environmental (EE) housing and short-term isolation conditions. The results demonstrated a significant increase in cldn5 and tjp levels in the chronically isolated group. Despite some anomalous results, alterations in mRNA levels were further confirmed in EE housing conditions compared with chronically isolated rats. This trend was also observed in rats subjected to short-term isolation compared with paired controls. Additionally, levels of IL-6, an inflammatory marker associated with neuroinflammation, were markedly increased in the isolated group. However, treatment with fluoxetine treatment reversed these effects. The results indicated that BBB
\end{abstract}

Correspondence to: Dr Tahani K. Alshammari, Department of Pharmacology and Toxicology, College of Pharmacy, King Saud University, P.O. Box 2457, Riyadh 11451, Kingdom of Saudi Arabia E-mail: talshammary@ksu.edu.sa

Key words: blood brain barrier, depression, socially isolated rats, claudin-5, tight junction, neuroinflammation integrity may be compromised in stress-related disorders, highlighting a need for further functional studies on the kinetics of BBB in stress-related models.

\section{Introduction}

Stress serves a primary role in the pathogenesis of psychiatric disorders (1). Previous studies have demonstrated that stress may eventually trigger or exacerbate mood disorders (2-4). Exposure to stress has profound consequences on physiological, biochemical and neurobehavioral function $(5,6)$.

Environmentally induced depression, such as chronic social isolation, has long been implicated as a risk factor for depression in humans and also induces anxiety and depression-like behavior in rodents. To date, environmental models are most commonly used for studying depression $(7,8)$.

Although depression and anxiety are highly prevalent serious stress-related psychiatric disorders, they are poorly understood $(9,10)$. In Saudi Arabia, the overall prevalence of depression has been reported to be $\sim 12 \%$ (11). Current treatment strategies have several major limitations, demonstrating the need to investigate pathological mechanisms and thus determine the most effective treatment strategy.

The blood-brain-barrier (BBB) is composed of endothelial cellular units and astrocytes interconnected by tight junction proteins. The integrity of BBB vascularity relies on the function of these tight junctions. The tight junction unit consists of various proteins, including claudin-5 (Cldn5) and certain tight junction proteins, such as tight junction protein 1 (TJP1). These are critical components that modulate BBB permeability (12). They are affected in multiple psychiatric and neurological diseases such as depression, Alzheimer's and other neurodegenerative disorders, brain trauma, stroke and multiple sclerosis (13)

A previous study demonstrated that cldn5 levels were decreased in the nucleus accumbens in depressed model rats. Furthermore, the introduction of a Cldn5 adeno-associated virus adeno-associated virus delivering shRNA against $c l d n 5$ in different brain regions increased the passage of peripheral IL-6 into the central nervous system (CNS), leading to depression-like behavior (14).

In a pharmacological model of depression (lipopolysaccharide-injected mice), tight junction proteins, including cldn5 
and tip, were significantly reduced. These results indicated that BBB dysfunction is associated with the dysregulation of ion transport, homeostasis and the passage of immune cells into the CNS (13). A widely recognized hypothesis is that of inflammation and depression. This theory explains the relationship between immune system function and its contribution to the neurobiology of depression $(15,16)$. It was previously reported that chronically isolated rats exhibited depressive-like behavior and, at a molecular level, multiple members of the Toll-like receptor (TLR) family were increased in the hippocampus (17).

Accumulating evidence has uncovered an association between mood disorders, particularly depression, and neuroinflammation (18). Clinical and preclinical studies have suggested that alterations in IL-6 levels are fundamental in the provocation of depression (19-21).

The present study aimed to: i) Examine the impact of stressful chronic social isolation on IL-6 levels in the hippocampal region of the brain; ii) investigate the mRNA expression of BBB markers in the hippocampus; iii) analyze the effect of acute fluoxetine treatment, a standard antidepressant; and iv) analyze the mRNA expression of BBB markers in different environmental conditions, such as an enriched environment and short-term isolation. To address these aims, the current study utilized different environmental conditions and pharmacological treatments.

\section{Materials and methods}

Animals. A total of 46 adult, 5-7 weeks of age, male Wistar rats (150-175 g) were obtained from the Animal Care Centre at the College of Pharmacy, King Saud University (Riyadh, Saudi Arabia). Animals were housed under a 12-h light/dark cycle at a temperature of $25 \pm 1^{\circ} \mathrm{C}$, with ad libitum access to food and water. Rats were left to adapt to the laboratory environment for 1 week prior to experimentation and were randomly divided into three main experiments. All experiments were carried out in accordance with the recommendations of the Experimental Animals Ethics Committee Acts of King Saud University, The Research Ethics Committee (approval no. KSU-SE-18-20).

Experiment one. For a 6-week period, rats were divided into four groups as follows ( $\mathrm{n}=10)$ : i) Paired ii) isolated; iii) isolated with fluoxetine treatment; and iv) paired with fluoxetine treatment. Fluoxetine $(25 \mathrm{mg} / \mathrm{kg}$ p.o.) was administered to isolated and paired rats on the 6th week (22) and to minimize stress during the experimental procedure, fluoxetine was administered in drinking water (17). The acutely treated group was used to examine the effects of short-term treatment. The paired-treated group served as a control. Antidepressants are known to significantly alter synaptic plasticity as these agents massively modulate multiple pathways and physiological mechanisms. They influence mood-related circuits, adult neurogenesis, neuronal survival, resiliency and adaptability (23).

After euthanizing the animals by $\mathrm{CO}_{2}$, using up to $30 \%$ displacement rate (approximately $5 \mathrm{l} / \mathrm{min}$ ), and the absence of reflexes verified death, trunk body blood was collected and brains were rapidly removed, snap-frozen in liquid nitrogen and stored at $-80^{\circ} \mathrm{C}$ until further use. Molecular changes in levels of BBB inflammatory markers (cldn5 and tjpI) and the central inflammatory marker (IL-6) were examined via reverse transcription-quantitative PCR (RT-qPCR) analysis. Levels of corticosterone, a peripherally stress-related marker, were additionally determined using ELISA.

Experiment two: Enriched environment (EE) housing as described previously (17). Parallel with experiment one, experiment two, involving EE conditions, was conducted. EE housing criteria was selected to further our understanding of the effect of environmental conditions on the molecular expression of BBB parameters at the mRNA level. A total of 10 rats were housed in a cage with dimensions of $1.5 \times 0.5 \times 0.7 \mathrm{~m}$. Bedding was changed every day for a 6 -week period. The animals were also provided with 8-10 toys, which were removed and washed three times a week, at which point half were then changed (22).

Experiment three: Short-term isolation as described previously (24). A total of 6 rats were divided into two groups (each, $n=3$ ). The first group was housed in standard conditions with three animals per cage. The second group had one rat per cage, where animals were housed for a total of 5 days. Rats were then sacrificed.

Serum corticosterone level determination. A trunk blood sample from each sacrificed rat was collected in regular tubes. Samples were then centrifuged at $30,588 \mathrm{xg}$ at $4^{\circ} \mathrm{C}$ for $30 \mathrm{~min}$ to obtain serum. After serum was collected in Eppendorf tubes, samples were stored at $-80^{\circ} \mathrm{C}$. Serum corticosterone levels were analyzed using an ELISA kit in accordance with the manufacturer's protocol (Abcam; cat. no. ab108821). The absorbance of the standards and samples was measured using a BioTek ${ }^{\circledR}$ Synergy ${ }^{\mathrm{TM}}$ HT microplate reader (Bio-Tek Instruments, Inc.) at a wavelength of $450 \mathrm{~nm}$.

Quantification of mRNA using RT-qPCR. RT-qPCR was conducted as described previously (24). Isolated hippocampal RNA was purified and converted to cDNA using a High-Capacity cDNA Reverse Transcription kit in accordance with the manufacturer's protocol. The following primers were utilized: IL-6 forward, 5'-CTTCCTAAAGATGGCTGCACT A-3' and reverse, 5'-CTGACTTGGCAGAGGACAAA-3'; cldn5 forward, 5'-AGCCCGCGTTCGGAAA-3' and reverse, 5'-ATTCAGCGGTGGTCGTCATC-3'; tjpl forward, 5'-CGA GGCATCGTTCCTAATAAGAA-3' and reverse, 5'-ATC GCCACCTGCTGTCTTTG-3'; GAPDH forward, 5'-GAC ATGCCGCCTGGAGAAAC-3' and reverse, 5'-AGCCCA GGATGCCCTTTAGT-3'. RT-qPCR analysis was conducted using SYBR Green based detection (Applied Biosystems 7500 QPCR detection system) with 7500 software (version 2.0.1) in accordance with the supplier's recommendations (each, Applied Biosystems; Thermo Fisher Scientific, Inc.). The relative expression of target mRNA was computed from the target cycle threshold (CT) value and the GAPDH CT value using the quantitative comparative $\mathrm{CT}(\Delta \Delta \mathrm{CT})$ method. These normalized values were then used to calculate a value expressing the fold change of the gene relative to the control according to the Livak method. The RT-qPCR was set up as follows: $50^{\circ} \mathrm{C}$ for $2 \mathrm{~min}, 95^{\circ} \mathrm{C}$ for $10 \mathrm{~min}$, then 40 cycles: $95^{\circ} \mathrm{C}$ for $15 \mathrm{sec}, 60^{\circ} \mathrm{C}$ for $1 \mathrm{~min}(25)$. 


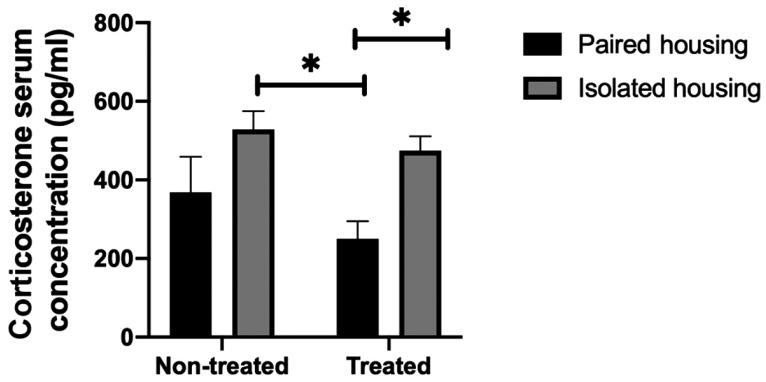

Figure 1. Determination of serum corticosterone levels in paired, chronically isolated, treated paired and treated isolated rats. Data were analyzed using two-way ANOVA followed by Tukey's multiple comparisons post hoc test and expressed as $(\mathrm{ng} / \mathrm{ml})$. Data are presented as the mean \pm SEM $(\mathrm{n}=5-6$ per group). ${ }^{*} \mathrm{P}<0.05$.

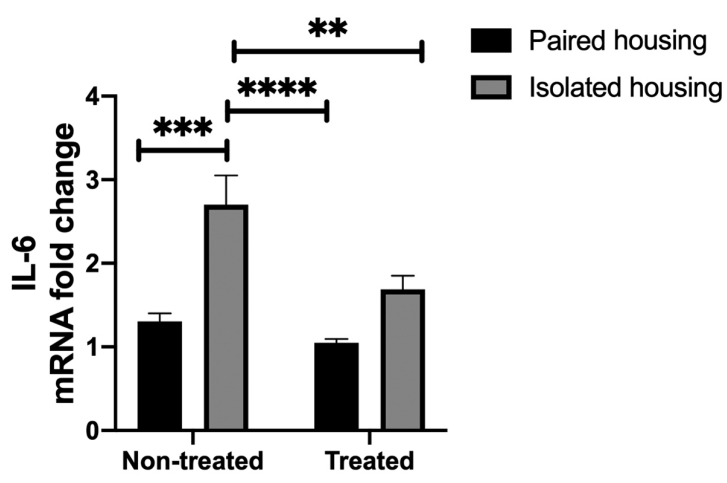

Figure 2. Central expression of inflammatory markers in the experimental groups. The mRNA expression levels of IL- 6 in the hippocampus of the tested groups were determined by reverse transcription-quantitative PCR analysis with GAPDH as an internal control. Data are expressed as fold change. Data are presented as the mean \pm SEM ( $n=7$ per group). Data were analyzed using a one-way ANOVA followed by a Tukey-Kramer post hoc test. ${ }^{* *} \mathrm{P}<0.01,{ }^{* * *} \mathrm{P}<0.001$ and ${ }^{* * * *} \mathrm{P}<0.0001$. IL-6, interleukin 6 .

Statistical analysis. All statistical analyses were conducted using GraphPad Prism version 8 (GraphPad Software, Inc.). Differences between two groups were determined using an unpaired Student's t-test and a Mann-Whitney U-test. Differences between paired isolated, isolated + fluoxetine and paired + fluoxetine groups were determined using two-way ANOVA followed by Tukey's multiple comparisons post hoc tests ( $\alpha$ level, 0.05 ). Data are presented as the mean \pm SEM and $\mathrm{P}<0.05$ was considered to indicate a statistically significant difference.

\section{Results}

Corticosterone levels in the periphery. As corticosterone is an indicator of stress, the present study examined serum levels in paired, chronically isolated, treated paired and isolated rats using two-way ANOVA followed by a Tukey post hoc test. Treatment by housing condition interaction was not significant $\left(\mathrm{F}_{1,17}=4.689 ; \mathrm{P}=0.5809\right)$; however the overall effect of housing conditions was significant $\left(\mathrm{F}_{1,17}=11.37 ; \mathrm{P}=0.0036\right)$. Serum corticosterone levels in the chronically isolated group were increased when compared with the paired group. Furthermore, a significant difference between the levels of serum corticosterone in chronically isolated rats and paired rats treated with fluoxetine was determined $(\mathrm{P}=0.0168$; determined using a
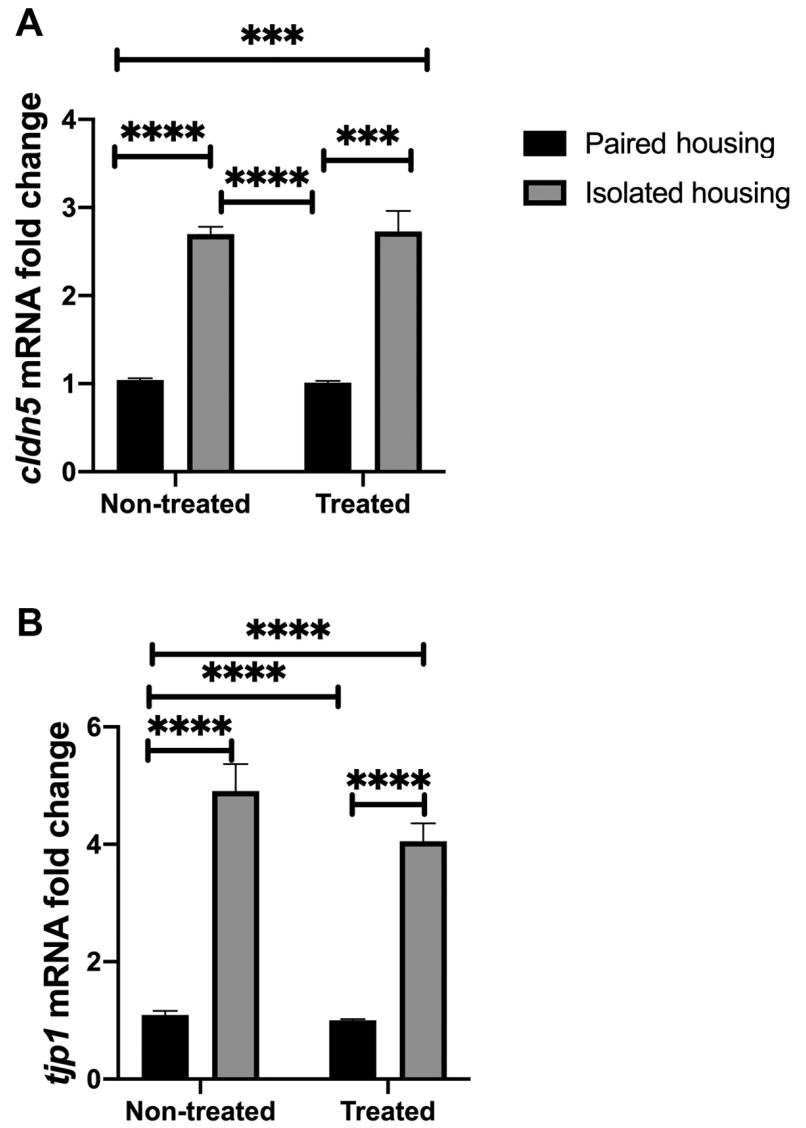

Figure 3. mRNA levels of BBB markers in the experimental groups. The expression of (A) cldn5 and (B) tjpl mRNA were determined. Data are expressed as fold change and were normalized to the paired control and paired housed rats. Dara are presented as the mean \pm SEM ( $n=7-8$ per group). Data were analyzed using a one-way ANOVA. ${ }^{* * *} \mathrm{P}<0.001$ and ${ }^{* * * *} \mathrm{P}<0.0001$ $\mathrm{BBB}$, blood-brain-barrier; cldn5, claudin-5; tjp1, tight junction protein 1 .

Tukey's post-hoc multiple comparisons test). Similarly, the level of serum corticosterone was significantly higher in isolated treated rats compared with paired treated rats $(\mathrm{P}=0.0498)$. The results indicated that the 1-week treatment with fluoxetine did not have a significant effect on serum corticosterone levels in the isolated groups compared with non-treated isolated groups ( $\mathrm{P}=0.9000$; Fig. 1).

Inflammatory mediator levels in the hippocampus of chronically isolated rats, isolated rats treated with fluoxetine and paired rats treated with fluoxetine. Levels of IL-6 in the hippocampus were assessed in the four tested groups. Two-way ANOVA indicated significant effects following housing $\left(\mathrm{F}_{1,24}=25.69 ; \mathrm{P}<0.0001\right)$ and treatment $\left(\mathrm{F}_{1,24}=9.960\right.$; $\mathrm{P}=0.0043)$; however, the interaction between the two was not significant $\left(\mathrm{F}_{1,24}=3.529 ; \mathrm{P}=0.0725\right)$. A significant increase in IL-6 mRNA levels were demonstrated in the chronic social isolation-induced group compared with the paired housing group $(\mathrm{P}=0.0003)$, as determined using a Tukey's post-hoc multiple comparisons test. Additionally, IL-6 mRNA was significantly increased in the hippocampi of isolated rats when compared with paired rats treated with fluoxetine $(\mathrm{P}<0.0001)$. Treatment with fluoxetine significantly reduced IL-6 levels in the isolated group compared with non-treated isolated rats ( $\mathrm{P}=0.0081$; Fig. 2). 
A

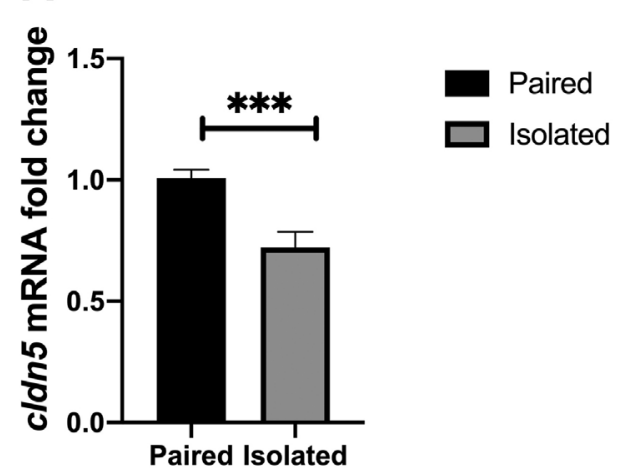

B

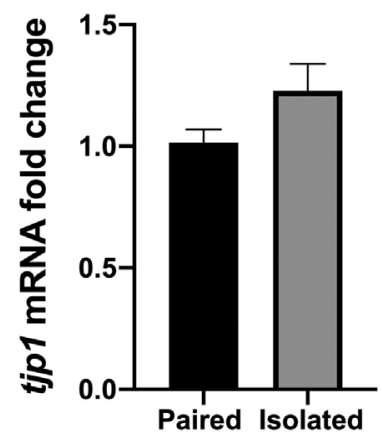

Figure 4. Expression of mRNA BBB markers following short term social isolation. The mRNA expression of BBB markers in the acute environmental isolated group was compared with that of the standard paired housed group. The mRNA expression of (A) cldn 5 and (B) tjpl was assessed in the hippocampus brain region of rats, as determined by RT-qPCR analysis with GAPDH as an internal control. Data are expressed as fold change and presented as the mean \pm SEM ( $n=10-12$ per group). Data were analyzed using a two-tailed unpaired t-test. ${ }^{* * *} \mathrm{P}<0.0001$. BBB, blood-brain-barrier; cldn5, claudin-5; tjp1, tight junction protein 1 .

Neurovascular integrity at the molecular level. The current study investigated whether alterations in tight junction proteins were associated with changes in BBB integrity at the mRNA level. To achieve this, the mRNA levels of cldn5 and tjpl were assessed in the four tested groups (paired, isolated, isolated + fluoxetine and paired +fluoxetine). Two-way ANOVA was utilized to quantify cldn5 mRNA expression. The results indicated no significance between tight junction proteins and BBB integrity. However, the effect of housing conditions was substantial $\left(\mathrm{F}_{1,27}=170.4 ; \mathrm{P}<0.0001\right)$. Tukey's post hoc analysis indicated that cldn $5 \mathrm{mRNA}$ expression was increased in both isolated and fluoxetine-treated isolated groups compared to the paired housed group $(\mathrm{P}<0.0001)$. Additionally, the expression of cldn 5 was significantly higher in the isolated group compared with the paired fluoxetine-treated group $(\mathrm{P}<0.0001)$. In each treated group, post hoc analysis revealed that $C l d n 5$ expression was significantly increased in the isolated group compared with the paired group $(\mathrm{P}<0.0001$; Fig. $3 \mathrm{~A})$. BBB in conditions of stress was further assessed by measuring tjpl, an additional BBB-related gene. tjpl acts as a tight junction adaptor protein that also regulates adherence junctions (26). Two-way ANOVA analysis indicated that the interaction between tjpl and treatment was not significant, while the effect of housing conditions was $\left(\mathrm{F}_{1,27}=170.4 ; \mathrm{P}<0.0001\right)$. Tukey's post hoc analysis indicated that tjpl mRNA expression was increased in the isolated

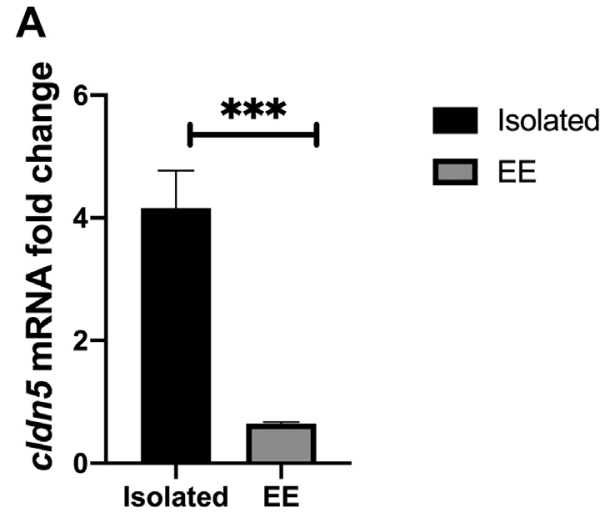

B

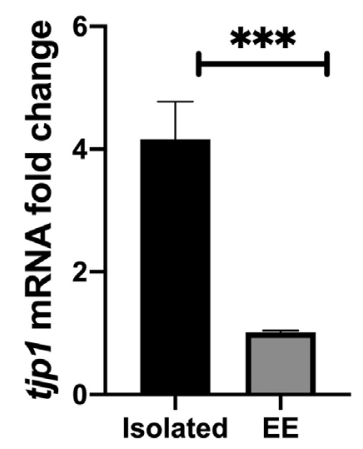

Figure 5. mRNA expression of the BBB in the enriched environmental (EE) group compared with the chronically isolated group. The mRNA expression of (A) cldn5 and (B) tjpl was assessed in the hippocampus brain region of rats, as determined by RT-qPCR analysis with GAPDH as an internal control. Data are expressed as fold change and presented as the mean \pm SEM ( $n=8-12$ per group). Data were analyzed using a two tailed unpaired t test. ${ }^{* * *} \mathrm{P}<0.0001$. BBB, blood-brain-barrier; cldn5, claudin-5; tjp1, tight junction protein 1.

and fluoxetine-treated isolated groups compared to the paired housed group $(\mathrm{P}<0.0001)$. Additionally, TJP1 expression was significantly higher in the isolated group compared with the paired fluoxetine-treated group $(\mathrm{P}<0.0001)$. In each treated group, post hoc analysis demonstrated that TJP1 expression was significantly increased in the isolated group compared with the paired group $(\mathrm{P}<0.0001$; Fig. 3B).

$B B B$ marker mRNA levels in different environmental conditions. The current study investigated whether BBB markers could be affected by two different environmental conditions. Short-term isolation was conducted using two main groups, a short-term isolated group and a standard paired housing group. Short-term isolation is considered a stressful condition that has been employed to address questions associated with mood disorders (27). The results of the present study indicated that cldn5 mRNA was decreased in the short-term isolation conditions compared with standard paired housed rats $(\mathrm{P}=0.0008$; Fig. 4A). Although tjpl mRNA levels were not affected by short term isolation, increased levels were observed in rats of the isolated housing group ( $\mathrm{P}>0.05$; Fig. 4B).

The second experimental setup comprised an EE approach. This was employed for 6 weeks, along with 6 weeks of social isolation. The results indicated that cldn5 mRNA levels were significantly reduced in EE compared with chronically isolated rats $(\mathrm{P}=0.0004$; Fig. $5 \mathrm{~A})$. The results suggested that 
there was a significant reduction in the level of tjpl mRNA in the EE group compared with the chronically isolated group $(\mathrm{P}=0.0006$; Fig. 5B).

\section{Discussion}

The present study determined that hippocampal IL-6 levels were increased in rats experiencing chronic social isolation. Additionally, the mRNA levels of certain blood-brain-barrier (BBB) markers, including claudin-5 (cldn5) and tight junction protein (tjpl), were increased in the isolated groups. The vulnerability of BBB markers was determined at the mRNA level in short-term isolated rats and in those under enriched environmental (EE) conditions. The results of the present study supports existing research $(14,28)$, which furthers our understanding regarding the complexity of pathological changes affecting BBB integrity and the level of inflammation in stress-related disorders.

Social isolation has been linked to the development of anxiety and depressive-like behaviors in rodents and depression in humans $(17,24,29)$. Different environmental approaches were utilized in the current study. EE housing is commonly used to investigate mechanisms associated with stress-related disorders and resilience $(2,30,31)$, while acute stress is a valid tool for the etiological examination of stress and stress coping mechanisms (24,32-34).

Under physiological conditions, the BBB serves a pivotal role in regulating molecular exchange between peripheral blood and the central nervous system (CNS). Proper maintenance of this perfusion homeostasis is essential. Aberrant structures and functions in endothelial subunits that constitute the $\mathrm{BBB}$ result in an inability to maintain adequate CNS perfusion. However, the pathological relevance of neurovascular dysfunction is poorly understood (35-37).

Changes in BBB integrity have been reported in various conditions such as poisoning, disruptions to the immune system and diabetes (38). Furthermore, in the context of neurological disorders, BBB permeability is altered in cases of traumatic brain and spinal cord injury, and in stroke patients (39). Furthermore, in a model of major depressive disorder, Menard et al (14) reported that Cldn5, a standard BBB marker, was altered. Li et al (40) also reported that connexin 40 , which is a gap junction protein and an indicator of astrocytic population general health, was altered in chronic mildly stressed rats. It was additionally determined that oral administration of fluoxetine reversed these changes. Similarly, postmortem studies have revealed that various members of the connexin family were altered in the brains of depressed, suicidal patients. These tight junction proteins were dysregulated at the mRNA level in brain regions such as the cerebellar cortex, thalamus and caudate nucleus (41). Taken together, this evidence supports the hypothesis of BBB dysfunction in depression.

The results of the current study demonstrated that chronic social isolation decreases BBB permeability, as determined by an increase in cldn5 and tjpl mRNA expression. Conditions of acute social isolation reduced the expression of cldn5 compared with the controls. However, the results also indicated a non-significant elevation in the mRNA levels of tjpl in the hippocampus. This result suggested that cldn 5 may be more sensitive and vulnerable to stressful social conditions. This could be a compensation mechanism that occurs in response to prolonged stressful isolation.

In contrast to the results of the present study, Menard et al (14) demonstrated that a model of chronic social defeat stress reduced the mRNA expression of cldn5 in the nucleus accumbens. Conversely, cldn 5 expression was elevated in the hippocampus of socially defeated rats at the protein level, suggesting a potential compensatory mechanism at the nucleic acid and protein level, as well as region-specific compensation. The discrepancy between these results may be attributed to the fact that the increased cldn 5 expression found in the current study was detected in the hippocampus, and different brain regions may have different responses. Another explanation is that different animal models might affect this mechanism in different ways. For example, the present study used an environmental isolation model, whereas Menard et al (14) used a social defeat model. Another marker is tjpl, which is an indispensable protein of the BBB. It is required for the appropriate assembly of tight junctions, which are pivotal to the interendothelial integrity of the BBB (42).

In contrast to previous research (14), the current study demonstrated that tjpl mRNA expression was increased in chronically isolated rats. This may be due to inflammatory mediators, such as TLR7, residing in close proximity to these elements of the BBB, leading to overall inflammation, swelling and junction closing. Previous studies have demonstrated that tjpl expression is reduced in patients with depression $(14,43)$.

$A$ reduction in $\mathrm{BBB}$ integrity leads to the infiltration of peripheral cytokines, including IL-6, into the brain, which affects neuronal populations and leads to observable depression-like behavior (14). Furthermore, tight junction proteins control the passage of macromolecules and ionic components in and out of the BBB and, as a consequence, regulate homeostasis $(44,45)$. The current study demonstrated that the administration of fluoxetine altered the permeability of the $\mathrm{BBB}$, indicating that social isolation alters BBB permeability and that acute pharmacological treatment with fluoxetine normalizes this effect.

A study by Fiorentino et al (46) demonstrated that, in the postmortem tissue of patients diagnosed with autism spectrum disorders, the BBB was disrupted. CLDN5 expression was elevated in different brain regions, including the cortex and cerebellum. Furthermore, these alterations were associated with a $66 \%$ increase in tight junction proteins, including claudin, in the intestines of these patients. This change in BBB integrity was coupled with peripheral inflammation. However, an elevation in BBB markers does not necessarily indicate that the protein produced is functional; in fact, these data could suggest that the CLDN5 protein produced in patients with autism may be disrupted or truncated, and that the body then provides additional mRNA to compensate.

Previous research revealed that patients with depression exhibit all the cardinal features of an inflammatory response. Peripheral blood gene expression profiles are consistent with an over-production of IL-6 and IL-8. Furthermore, the increased expression of a variety of innate immune genes and proteins, including IL-1 $\beta$, IL- 6 , TNF, TLR3 and TLR4, has been found in post-mortem brain samples from suicide victims with depression (16). IL-6 has been linked to stress-related 
disorders such as depression and anxiety. Many patients with major depressive disorder also have higher levels of IL-6 $(14,47,48)$. In rodents, both peripheral and hippocampal levels of IL-6 are increased. For example, 4 weeks of constant darkness used as a model of seasonal affective disorder, has been revealed to cause depression-like behavior in rodents. Moreover, levels of IL-6 were also altered (49). In line with this result, IL- 6 knockout mice have been found to be resistant to the development of a depression-like phenotype following exposure to constant darkness. This suggests a functional role for IL-6 in stress susceptibility $(47,49)$.

The results of the present study are similar to those of previous reports, with data indicating that exposure to a stressful environment leads to changes in the serum levels of corticosterone and inflammatory mediators, along with an increase in IL-6 levels of the brain. The current study provides evidence that, following exposure to stressful events (chronically and acutely isolated housing conditions), rats exhibited alterations in the levels of BBB mRNA expression in the hippocampus. Some of these changes were reversed by acute pharmacological treatment with fluoxetine. The present study emphasized the role of the BBB in the pathology of stress-related mood disorders. Future studies should examine the functional kinetics of BBB integrity and fully characterize the architecture of the BBB unit, which would aid in addressing whether elevations in mRNA levels reflect an increase in the expression of fully functional tight junction proteins. Future studies should also describe the expression and structure of other BBB components.

\section{Acknowledgements}

Not applicable.

\section{Funding}

No funding was received.

\section{Availability of data and materials}

The datasets used and/or analyzed during the current study are available from the corresponding author on request.

\section{Authors' contributions}

TKA designed the current study and contributed to the acquisition, analysis and interpretation of data. HMA conducted RT-qPCR and biochemical experiments and contributed to the analysis and interpretation of data. HEA wrote the manuscript and contributed to the analysis of the data. NMA, MAA and MFS provided intellectual support and contributed to the study design and drafting of the manuscript. All authors read and approved the final manuscript.

\section{Ethics approval and consent to participate}

All experiments were carried out in accordance with the recommendations of the Experimental Animals Ethics Committee Acts of King Saud University, The Research Ethics Committee (approval no. KSU-SE-18-20).

\section{Patient consent for publication}

Not applicable.

\section{Competing interests}

The authors declare that they have no competing interests.

\section{References}

1. Abelaira HM, Réus GZ and Quevedo J: Animal models as tools to study the pathophysiology of depression. Braz J Psychiatry 35 (Suppl 2): S112-S120, 2013.

2. Ilin Y and Richter-Levin G: Enriched environment experience overcomes learning deficits and depressive-like behavior induced by juvenile stress. PLoS One 4: e4329, 2009.

3. Fernández-Guasti A, Fiedler JL, Herrera L and Handa RJ: Sex, stress, and mood disorders: At the intersection of adrenal and gonadal hormones. Horm Metab Res 44: 607-618, 2012.

4. Calabrese F, Molteni R, Racagni G and Riva MA: Neuronal plasticity: A link between stress and mood disorders. Psychoneuroendocrinology 34 (Suppl 1): S208-S216, 2009.

5. Segerstrom SC and Miller GE: Psychological stress and the human immune system: A meta-analytic study of 30 years of inquiry. Psychol Bull 130: 601-630, 2004.

6. Lupien SJ, McEwen BS, Gunnar MR and Heim C: Effects of stress throughout the lifespan on the brain, behaviour and cognition. Nat Rev Neurosci 10: 434-445, 2009.

7. Cacioppo JT, Cacioppo S, Capitanio JP and Cole SW: The neuroendocrinology of social isolation. Annu Rev Psychol 66: 733-767, 2015.

8. Coleman K, Weed JL and Schapiro SJ: Environmental enrichment for animals used in research. In: Animal Models for the Study of Human Disease. Conn PM (ed). Academic Press, London, pp74-94, 2013.

9. Jia J and Le W: Molecular network of neuronal autophagy in the pathophysiology and treatment of depression. Neurosci Bull 31: 427-434, 2015.

10. Mehta-Raghavan NS, Wert SL, Morley C, Graf EN and Redei EE: Nature and nurture: Environmental influences on a genetic rat model of depression. Transl Psychiatry 6: e770, 2016.

11. Al-Qadhi W, Ur Rahman S, Ferwana MS and Abdulmajeed IA: Adult depression screening in Saudi primary care: Prevalence, instrument and cost. BMC Psychiatry 14: 190, 2014.

12. Jiao H, Wang Z, Liu Y, Wang P and Xue Y: Specific role of tight junction proteins claudin-5, occludin, and $\mathrm{ZO}-1$ of the blood-brain barrier in a focal cerebral ischemic insult. J Mol Neurosci 44: 130-139, 2011.

13. Daneman R and Prat A: The blood-brain barrier. Cold Spring Harb Perspect Biol 7: a020412, 2015.

14. Menard C, Pfau ML, Hodes GE, Kana V, Wang VX, Bouchard S, Takahashi A, Flanigan ME, Aleyasin H, LeClair KB, et al: Social stress induces neurovascular pathology promoting depression. Nat Neurosci 20: 1752-1760, 2017.

15. Haapakoski R, Ebmeier KP, Alenius $\mathrm{H}$ and Kivimäki M: Innate and adaptive immunity in the development of depression: An update on current knowledge and technological advances. Prog Neuropsychopharmacol Biol Psychiatry 66: 63-72, 2016.

16. Miller AH and Raison CL: The role of inflammation in depression: From evolutionary imperative to modern treatment target. Nat Rev Immunol 16: 22-34, 2016.

17. Alshammari TK, Alghamdi H, Green TA, Niazy A, Alkahdar L, Alrasheed N, Alhosaini K, Alswayyed M, Elango R, Laezza F, et al: Assessing the role of toll-like receptor in isolated, standard and enriched housing conditions. PLoS One 14: e0222818, 2019.

18. Liu YZ, Wang YX and Jiang CL: Inflammation: The common pathway of stress-related diseases. Front Hum Neurosci 11: 316, 2017.

19. Hodes GE, Ménard C and Russo SJ: Integrating Interleukin-6 into depression diagnosis and treatment. Neurobiol Stress 4: 15-22, 2016.

20. Ting EY, Yang AC and Tsai SJ: Role of interleukin-6 in depressive disorder. Int J Mol Sci 21: 2194, 2020.

21. Himmerich H, Patsalos O, Lichtblau N, Ibrahim MAA and Dalton B: Cytokine research in depression: principles, challenges, and open questions. Front Psychiatry 10: 30, 2019. 
22. Moncek F, Duncko R, Johansson BB and Jezova D: Effect of environmental enrichment on stress related systems in rats. J Neuroendocrinol 16: 423-431, 2004.

23. Castrén E and Hen R: Neuronal plasticity and antidepressant actions. Trends Neurosci 36: 259-267, 2013.

24. Alshammari TK, Alghamdi H, Alkhader LF, Alqahtani Q, Alrasheed NM, Yacoub H, Alnaem N, AlNakiyah M and Alshammari MA: Analysis of the molecular and behavioral effects of acute social isolation on rats. Behav Brain Res 377 112191, 2020.

25. Livak KJ and Schmittgen TD: Analysis of relative gene expression data using real-time quantitative PCR and the 2(-Delta Delta C(T)) method. Methods 25: 402-408, 2001.

26. Bauer HC, Krizbai IA, Bauer H and Traweger A: 'You Shall Not Pass'-tight junctions of the blood brain barrier. Front Neurosci 8 : 392, 2014.

27. Holt-Lunstad J: The potential public health relevance of social isolation and loneliness: Prevalence, epidemiology, and risk factors. Public Policy Aging Rep 27: 127-130, 2017.

28. Dudek KA, Dion-Albert L, Lebel M, LeClair K, Labrecque S Tuck E, Ferrer Perez C, Golden SA, Tamminga C, Turecki G, et al Molecular adaptations of the blood-brain barrier promote stress resilience vs. depression. Proc Natl Acad Sci USA 117: 3326-3336, 2020.

29. Sargin D, Oliver DK and Lambe EK: Chronic social isolation reduces 5-HT neuronal activity via upregulated SK3 calcium-activated potassium channels. Elife 5: e21416, 2016.

30. Lehmann ML and Herkenham M: Environmental enrichment confers stress resiliency to social defeat through an infralimbic cortex-dependent neuroanatomical pathway. J Neurosci 31 6159-6173, 2011.

31. McCreary JK and Metz GAS: Environmental enrichment as an intervention for adverse health outcomes of prenatal stress. Environ Epigenet 2: dvw013, 2016.

32. Takatsu-Coleman AL, Patti CL, Zanin KA, Zager A, Carvalho RC, Borçoi AR, Ceccon LM, Berro LF, Tufik S, Andersen ML and Frussa-Filho R: Short-term social isolation induces depressive-like behaviour and reinstates the retrieval of an aversive task: Mood-congruent memory in male mice? J Psychiatry Neurosci 38: 259-268, 2013.

33. de Kloet ER, Joëls M and Holsboer F: Stress and the brain: From adaptation to disease. Nat Rev Neurosci 6: 463-475, 2005.

34. Kim JW, Ko MJ, Gonzales EL, Kang RJ, Kim DG, Kim Y, Seung H, Oh HA, Eun PH and Shin CY: Social support rescues acute stress-induced cognitive impairments by modulating ERK1/2 phosphorylation in adolescent mice. Sci Rep 8: 12003, 2018.

35. Quaegebeur A, Lange $C$ and Carmeliet $P$ : The neurovascular link in health and disease: Molecular mechanisms and therapeutic implications. Neuron 71: 406-424, 2011.

36. Rubio-AraizA,PorcuF,Pérez-HernándezM,García-GutiérrezMS, Aracil-Fernández MA, Gutierrez-López MD, Guerri C, Manzanares J, O'Shea E and Colado MI: Disruption of blood-brain barrier integrity in postmortem alcoholic brain: Preclinical evidence of TLR4 involvement from a binge-like drinking model. Addict Biol 22: 1103-1116, 2017.
37. Wolburg H, Noell S, Mack A, Wolburg-Buchholz K and Fallier-Becker P: Brain endothelial cells and the glio-vascular complex. Cell Tissue Res 335: 75-96, 2009.

38. Venkat P, Chopp M and Chen J: Blood-brain barrier disruption, vascular impairment, and ischemia/reperfusion damage in diabetic stroke. J Am Heart Assoc 6: e005819, 2017.

39. Chodobski A, Zink BJ and Szmydynger-Chodobska J: Blood-brain barrier pathophysiology in traumatic brain injury. Transl Stroke Res 2: 492-516, 2011.

40. Li DQ, Li XJ, Duan JF and Cai W: Wuling Capsule promotes hippocampal neurogenesis by improving expression of connexin 43 in rats exposed to chronic unpredictable mild stress. Zhong Xi Yi Jie He Xue Bao 8: 662-669, 2010.

41. Nagy C, Torres-Platas SG, Mechawar N and Turecki G: Repression of astrocytic connexins in cortical and subcortical brain regions and prefrontal enrichment of $\mathrm{H} 3 \mathrm{~K} 9 \mathrm{me} 3$ in depression and suicide. Int J Neuropsychopharmacol 20: 50-57, 2017.

42. Bauer $H$, Zweimueller-Mayer $J$, Steinbacher $P$, Lametschwandtner A and Bauer HC: The dual role of zonula occludens (ZO) proteins. J Biomed Biotechnol 2010: 402593, 2010.

43. Esposito P, Gheorghe D, Kandere K, Pang X, Connolly R, Jacobson $S$ and Theoharides TC: Acute stress increases permeability of the blood-brain-barrier through activation of brain mast cells. Brain Res 888: 117-127, 2001.

44. Greene $\mathrm{C}$ and Campbell M: Tight junction modulation of the blood brain barrier: CNS delivery of small molecules. Tissue Barriers 4: e1138017, 2016.

45. Stamatovic SM, Keep RF and Andjelkovic AV: Brain endothelial cell-cell junctions: how to 'open' the blood brain barrier. Curr Neuropharmacol 6: 179-192, 2008.

46. Fiorentino M, Sapone A, Senger S, Camhi SS, Kadzielski SM, Buie TM, Kelly DL, Cascella N and Fasano A: Blood-brain barrier and intestinal epithelial barrier alterations in autism spectrum disorders. Mol Autism 7: 49, 2016.

47. Hodes GE, Kana V, Menard C, Merad M and Russo SJ Neuroimmune mechanisms of depression. Nat Neurosci 18: 1386-1393, 2015

48. Ménard C, Hodes GE and Russo SJ: Pathogenesis of depression: Insights from human and rodent studies. Neuroscience 321: 138-162, 2016.

49. Monje FJ, Cabatic M, Divisch I, Kim EJ, Herkner KR, Binder BR and Pollak DD: Constant darkness induces IL-6-dependent depression-like behavior through the NF- $\mathrm{kB}$ signaling pathway. J Neurosci 31: 9075-9083, 2011.

This work is licensed under a Creative Commons Attribution-NonCommercial-NoDerivatives 4.0 International (CC BY-NC-ND 4.0) License. 